\title{
Article \\ Modeling Random Exit Selection in Intercity Expressway Traffic with Quantum Walk
}

\author{
Dongshuang $\mathrm{Li}^{1,2}, \mathrm{Xu} \mathrm{Hu}^{3}$, Xinxin Zhou ${ }^{4,5}$, Wen Luo ${ }^{3,6}$, A. Xing $\mathrm{Zhu}^{7}$ and Zhaoyuan $\mathrm{Yu}^{3,6, *(1)}$ \\ 1 Jiangsu Key Laboratory of Crop Genetics and Physiology/Jiangsu Key Laboratory of Crop Cultivation \\ and Physiology, Agricultural College of Yangzhou University, Yangzhou 225009, China; 007250@yzu.edu.cn \\ 2 Jiangsu Co-Innovation Center for Modern Production Technology of Grain Crops, Yangzhou University, \\ Yangzhou 225009, China \\ 3 Key Laboratory of Virtual Geographic Environment, Nanjing Normal University, Ministry of Education, \\ Nanjing 210023, China; 191302068@njnu.edu.cn (X.H.); 09415@njnu.edu.cn (W.L.) \\ 4 School of Geographic and Biologic Information, Nanjing University of Posts and Telecommunications, \\ Nanjing 210023, China; zhouxinxin@njupt.edu.cn \\ 5 Smart Health Big Data Analysis and Location Services Engineering Research Center of Jiangsu Province, \\ Nanjing University of Posts and Telecommunications, Nanjing 210023, China \\ 6 Jiangsu Center for Collaborative Innovation in Geographical Information Resource Development and \\ Application, Nanjing 210023, China \\ 7 Department of Geography, University of Wisconsin-Madison, Madison, WI 53705, USA; azhu@wisc.edu \\ * Correspondence: yuzhaoyuan@njnu.edu.cn
}

Citation: Li, D.; Hu, X.; Zhou, X.; Luo, W.; Zhu, A.X.; Yu, Z. Modeling Random Exit Selection in Intercity Expressway Traffic with Quantum Walk. Appl. Sci. 2022, 12, 2139. https://doi.org/10.3390/app12042139

Academic Editor: Paola Pellegrini

Received: 9 November 2021

Accepted: 26 January 2022

Published: 18 February 2022

Publisher's Note: MDPI stays neutral with regard to jurisdictional claims in published maps and institutional affiliations.

Copyright: (C) 2022 by the authors. Licensee MDPI, Basel, Switzerland. This article is an open access article distributed under the terms and conditions of the Creative Commons Attribution (CC BY) license (https:// creativecommons.org/licenses/by/ $4.0 /)$.

\begin{abstract}
In intercity expressway traffic, the multiplicity of available routes leads to randomness in exit selection. Random exit selection by drivers is hard to observe, and thus it is a challenge to model intercity expressway traffic sufficiently. In this paper, we developed a Random Quantum Traffic Model (RQTM), which modeled the stochastic traffic fluctuation caused by random exit selection and the residual regularity fluctuation with the quantum walk and autoregressive moving average model (ARMA), respectively. The RQTM considered the random exit selection of a driver as a quantum stochastic process with a dynamic probability function. A quantum walk was applied to update the probability function, which simulated when and where a driver will leave the expressway. We validated our model with hourly traffic data from seven exits from the Nanjing-Changzhou expressway in eastern China. For the seven exits, the coefficients of determination of the RQTM ranged from 0.5 to 0.85 . Compared with the classical random walk and the ARMA model, the coefficients of determination were increased by $21.28 \%$ to $104.98 \%$, and the relative mean square error decreased by $11.61 \%$ to $32.92 \%$. We conclude that the RQTM provides new potential for modeling traffic dynamics with consideration of unobservable random driver decision making.
\end{abstract}

Keywords: expressway traffic flow; traffic simulation; quantum walk; random exit selection

\section{Introduction}

When a driver heads to his/her destination via intercity expressways, despite the fact that the destination is often deterministic, the multiplicity of available routes leads to randomness in exit selection. Concretely, a driver will pick the travel route from multiple candidates based on considerations of economy, timeliness, and traffic conditions [1]. Under such a scenario, the exit section is randomly induced by route selection, contrary to the conventional deterministic exit selection. We call the random exit selection phenomenon route-induced random exit selection. The aggregation of the random exit selection by individual drivers will affect the distributions of traffic flow in space-time along the expressway [2]. Besides, due to the dense traffic flow and the relatively fast speed of the expressway traffic, any vehicle that passes through the expressway exit will affect the speed of the following vehicles. The combined effect of a large number of individual random decisions at different destinations/exits along the expressway makes the overall traffic 
flow exhibit significant inhomogeneities, nonlinearities, and uncertainties in space-time [3]. Considering this random exit selection of drivers in traffic flow simulation might help to reduce model uncertainty and improve model accuracy.

Current traffic volume simulation models can be divided into two major categories: deterministic and stochastic models [4]. Most of the current traffic volume simulation models consider the overall traffic volume and rarely consider the influence of random exit selection in the model. The deterministic models consider the overall traffic volume that can be accurately modeled and solved by numerical methods, and the output of the model is fully determined by the parameter values and the initial conditions. Deterministic models can be further classified into three subcategories: macroscopic models, microphysical models, and intelligent agent models [5]. Macroscopic models consider the overall effects of random exit selection. Such models commonly use endogenous or exogenous variables to summarize the effects for the traffic volumes at each exit, including the exit selections (e.g., origin-destination models [6] and logit analysis [7]). Therefore, it only reflects the total or average effects of traffic volume rather than the random exit selection of individual drivers [8]. Microphysical models (e.g., gravity models [9] and lagrangian traffic state models [10]) use physics laws to approximately describe the influences of random exit selection. Such models abstract each driver into indistinguishable particles, characterizing the influences of random exit selection by varying the attractiveness of the particles to approximate the spatial and temporal dynamics of each driver through different exits [11]. The intelligent agent models simulate the traffic volume from each exit through an agent with active sensing and decision-making ability [12]. Such models can support the random exit selection of drivers by giving the agent predefined evolution rules or targets to evolve the agent autonomously [13]. However, the predefined rules can only be used to model exits choices from the perspective of certainty rather than stochasticity [14]. Therefore, the intelligent agent models cannot reveal the impact of the dynamic nature of a driver's random exit selection in driving. These deterministic models have the advantages of having clear mechanisms and can be efficiently solved numerically, but they do not have the ability to model the dynamic effects of the random nature of drivers.

The second category is the stochastic models. Such models assume that random exit selection is a complex stochastic system, formed randomly under the influence of many factors and cannot be accurately modeled with simple deterministic physics or evolutionary rules. Stochastic models formulate random exit selection within a complex network, which considers each choice the driver made as a node in the network. Stochastic models commonly use a classical random walk to generate random paths that transit from one node to others to simulate the dynamic statistical characteristics of the drivers' random exit selection [15]. The classical random walk assumes that the transition probability between different nodes follows a particular probability distribution, thus the dynamics of all the traffic can be evaluated according to the statistical characteristics of the walk paths on the network [16-18]. Recently, with the continuous accumulation and refinement of traffic observation data [19-21], growing evidence shows that the classical random walk can well capture some nonlinear and nonstationary characteristics, e.g., power-law distribution [22], dynamic rerouting behavior [23], and percolation patterns [24] of urban traffics. However, rare study reports good results on applying the classical random walk on expressway traffic. This is because in the classical random walk, each driver leaving the expressway is independent. However, in expressway traffic flow, a driver's departure from the traffic flow during the exit selection process causes the state of the traffic flow to change, thereby affecting the random exit selection of the drivers in the subsequent traffic flow $[25,26]$.

There are several key challenges for the current models to integrate the effects of stochastic fluctuation caused by the random exit selection. Firstly, in a single random exit selection, the driver at a given time may consider exiting the expressway at multiple exits, which can occur at different times [22]. With this simultaneous decision making, the process of random exit selection for multiple alternatives should be modeled by a process of simultaneous searching and traversing of multiple alternative paths rather than a single 
path in the entire decision network. These will form a dynamically changing probability distribution that will then affect the spatial-temporal locations of the individual drivers and then lead to the fluctuations of the overall traffic volumes. The modeling of such chain interaction is difficult. Secondly, the dynamic decisions of drivers during the driving process can hardly be observed. Even if the trajectory of a driver can be logged with video or other methods, the detailed mechanisms of how a driver makes random exit selection is unknown [27]. With this unobservable nature of random exit selection, the deterministic model can hardly be used. Lastly, there are bidirectional interactions between the random exit selection and traffic volumes. The aggregation of random exit selection by many drivers will change the traffic condition, yet, the change of traffic condition will also affect the random exit selection of each driver. Because a continuous dynamic observation of a driver's choices of exits is not possible, the mechanisms of this bidirectional driver-to-traffic interactions are not clear. From the above discussion, it is clear that modeling the effects of the dynamic nature of random exit selection by drivers on traffic conditions is still a challenge yet to be addressed adequately.

Quantum walk, is the quantum extension of the classical random walk, which is an ideal tool to solve dynamic and parallel stochastic path searching on complex networks $[28,29]$. In the quantum walk, when the system is not observed, the driver can choose any of the feasible nodes (exits) at the same time with different probabilities. The transition between the nodes on the network is not "either here or there" but "both here and there", thus the path generation in the quantum walk is "all possible paths" rather than "a single path" as in the classical random walk [30]. All possible paths then will collapse to a single determined path when the observation is applied. With efficient matrix algorithms [31], a quantum walk can converge much faster than the classical random walk [32]. Due to the advantages of the quantum walk, there are already many applications of the quantum walk in algorithm construction [33], decision-making simulation [34], and data analysis [35].

In this paper, we tried to overcome the above challenge of modeling and simulation of random exit selection by introducing the quantum walk models. By integrating the random exit selection from an interactive quantum probability perspective, we developed the Random Quantum Traffic Model (RQTM) to simulate the stochastic traffic fluctuation caused by random exit selection by a quantum walk (QW) model and the residual regularity fluctuation by an autoregressive moving average (ARMA) model. Our paper is organized as follows: Section 2 formulates the research problems. Section 3 introduces the overall modeling process. Section 4 presents a case study using the Nanjing-Changzhou Expressway with seven toll stations serving as validation. A discussion is provided in Section 5, and conclusions are drawn in Section 6.

\section{Problem Definition and Basic Idea}

\subsection{Formal Definition of the Problem}

Consider there is an expressway with $n$ different exits, there are in total $m$ drivers on the expressway at a specific time $T_{k}$. For each exit $E_{i}$, there is an observation station (e.g., toll station or video surveillance camera) $F_{i k}$, which is the total number of vehicles leaving the expressway through the exit $E_{i}$ at time $T_{k}$ with a sampling time interval $\Delta T_{k}$. Generally, we assumed all the $i, j, k$, which are the indexes of exit, driver, and time beginning from 1 , and $i=\{1,2, \cdots, n\}, j=\{1,2, \cdots, m\}$, and $k=\{1,2, \cdots, s\}$. In the real world, the only observable variable is $F_{i k}$, which is also one of the most important factors to indicate the characteristics of the expressway conditions. Obviously, $F_{i k}$ is continuously changing over time, and we hoped to simulate the dynamics of $F_{i k}$ as accurately as possible.

We assumed that $F_{i k}$ consists of two fundamental parts: the deterministic part $F_{i k d}$ and the random part $F_{i k r}$. We assumed that $F_{i k d}$ is a combination of completely predictable components, such as the mean value and the regular cycles of the traffic flow. Then $F_{i k r}$ 
is the unobservable random variation caused by random exit selection. So, we have the fundamental equation of traffic flow in each exit $E_{i}$ as

$$
F_{i k}=F_{i k d}+F_{i k r}
$$

As $F_{i k d}$ is totally predictable, which means we can use a simple model, such as the autoregressive moving average model, to approximate it. The major issue is how to simulate the dynamics of random exit selection to improve the predictability of $F_{i k r}$.

There were three basic procedures that were required to simulate $F_{i k r}$ by considering random exit selection. The first was how to model all the possible choices for exits as a random process, which we called the decision space. The second was modeling the probability distribution and its evolution to express the dynamic decision process on exit selection. The third was how to construct the relationship between the probability distribution and $F_{i k r}$. In the following sections, we will present our solution in modeling $F_{i k r}$ in three procedures. We first formulated the problem associated with each of these procedures and then described our basic idea in addressing these problems.

Procedure 1: Modeling all the possible choices of exits as the decision space. The essence of exit selection is that a driver chooses an exit to leave the expressway traffic at a specific time. Therefore, the key representation of the choice for a random exit is the presentation of a driver choosing which exit at what time with how much probability. Assuming that the decision process of any driver $D_{j}$ expected to exit the highway at exit $E_{i}$ at time $T_{k}$ is a node $V_{(i, k)}$, and the complete set of exit selections is $E_{i} \times T_{k}$. Therefore, in time and space, all possible exit selections of any driver $D_{j}$ can be combined into a tree-style network. Any node $V_{(i, k)}$ on the network is a state that a chosen exit, and any edge $E$ represents the spatiotemporal evolution of an exit selection of a given driver. As both the traffic direction and time of expressway traffic is in one direction, the path on the network can only be generated sequentially, therefore, we can reformulate the $n \times s$ network into a tree-style network as is shown in Figure 1. We named this tree-style network the exit selection decision space. For each node of this decision space, a probability vector can be assigned to express the driver's preference for the decision. Generally, an n-dimensional probability vector $P(i, j, k)=\left(p_{1}, p_{2}, \cdots, p_{n}\right)$, where $\left(p_{1}, p_{2}, \cdots, p_{n}\right)$ can be used to indicate the probability of a driver at any time $T_{k}$ to choose an exit $\left(E_{1}, E_{2}, \cdots, E_{i}\right)$ to exit traffic. Clearly, the probability vector $P(i, j, k)$ is unobservable.

Procedure 2: Modeling the probability evolution of exit selection. The dynamics of exit selection can be formulated as a dynamic path generation according to the dynamic change of the probability vector. Typically, the dynamic change of the probability vector can be expressed by the state transition matrix, namely,

$$
P(i, j, k)_{s 1}=T_{s 0 \rightarrow s 1} P(i, j, k)_{s 0}
$$

The full matrix expression form of Equation (2) is

$$
\left(\begin{array}{c}
p_{1}{ }^{\prime}(i, j, k) \\
\vdots \\
p_{n}{ }^{\prime}(i, j, k)
\end{array}\right)=\left(\begin{array}{ccc}
t_{11} & \cdots & t_{1 n} \\
\vdots & \ddots & \vdots \\
t_{n 1} & \cdots & t_{n n}
\end{array}\right) \cdot\left(\begin{array}{c}
p_{1}(i, j, k) \\
\vdots \\
p_{n}(i, j, k)
\end{array}\right)
$$

where $T_{s 0 \rightarrow s 1}=\left(\begin{array}{ccc}t_{11} & \ldots & t_{1 n} \\ \vdots & \ddots & \vdots \\ t_{n 1} & \cdots & t_{n n}\end{array}\right)$ is the state transition matrix, which reflects the transition process of random exit selection of a driver between different states.

In the real traffic flow process, not only the probability vector $P(i, j, k)_{s 0}$ and $P(i, j, k)_{s 1}$ but also the transition matrix $T_{s 0 \rightarrow s 1}$ are unobservable. As there are heterogonies in exit selection between different drivers, each driver has a specific transition matrix $T_{s 0 \rightarrow s 1}$. 


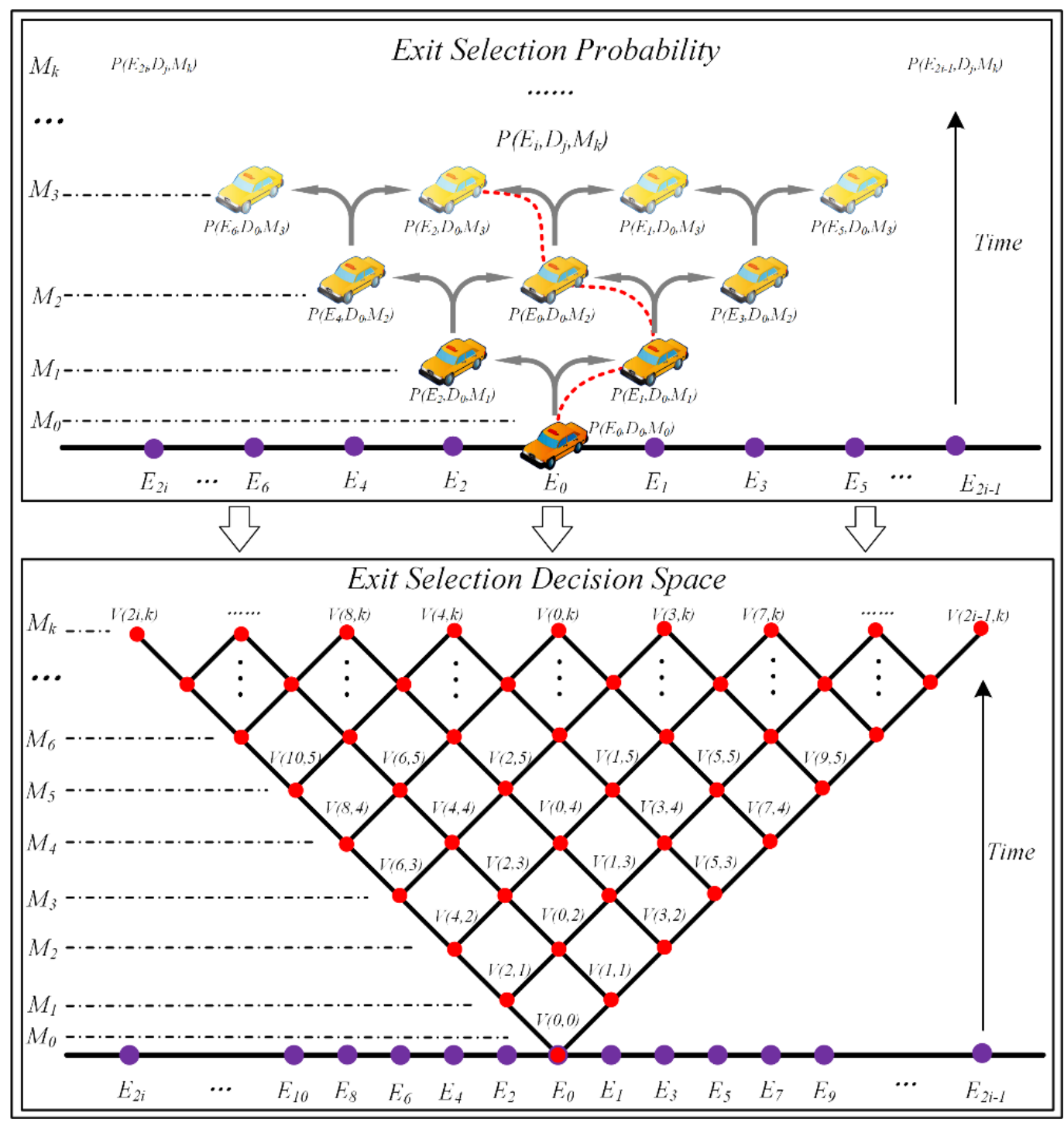

Figure 1. The quantum walk expressway traffic simulation model.

In real-world random exit selection, a driver indeed considers all the feasible choices as a total, which means in the network, he/she chooses every possible node available to generate multiple paths. These paths evolve through time, and the probability distribution of its position at any node is not deterministic. In addition, any changes in the selection of the probability of a node will affect all the probability distribution. As the probability is unobservable, the continuous change of probability will, in turn, create a chain reaction, which will then lead to a combinatorial explosion. This combinatorial explosion makes it difficult, if not impossible, to use deterministic or stochastic methods for both modeling and simulating the random exit selection of a massive number of drivers in expressway traffic.

Procedure 3: Mapping the dynamics of probability into real traffic volume. In order to simulate the real volume of traffic flow at each exit of the expressway, it is necessary to resolve the mapping between the dynamic of exit probability and the traffic flow at each exit. Since Procedure 2 only output the probability of whether a driver will choose a certain exit, a calibration is needed to map this probability to the real volume of the traffic flow. Since the observation of the state of each vehicle on the highway is not practical, the calibration and verification of the model can only be operated on the total amount of traffic observed at the exit. 
A generalized calibration model can be constructed as follows:

$$
F_{i k r}=f(P(i, k))
$$

where $F_{i k r}$ is the real traffic volume obtained by simulated probability, $P(i, k)$ is the simulated probability of leaving the expressway from exit $E_{i}$ at time $T_{k}$, and $f$ is the calibration function that maps $P(i, k)$ to $F_{i k r}$.

\subsection{Basic Idea}

The unobservable and combinatorial explosion nature of random exit selection, which are key difficulties to model under the classical statistical framework, can be modeled in the quantum walk. Unlike a classical random walk, which uses a probability vector that forces the driver to choose any node in the decision space of exit selection, the quantum walk assumes that at any time the driver will select all the possible choices, i.e., nodes, at a single time. The quantum walk uses a state vector, which is a vector that expresses how relatively a driver will choose each node at a time, to represent the decision making. With the state vector, a dynamic probability distribution with each node to show the preference of the driver can be generated. As the probability distribution of a state vector is unobservable at any time, the quantum walk chooses several decisions in parallel with the linear combination of the state vector (See details of the superposition in Section 3.1). It is more realistic in random exit selection that any driver will make a decision in parallel and select more than one choice at a single time. So, with the state vector representation, we can solve the unobservable probability problem in Procedure 1.

In the quantum walk, the characteristics of the dynamic path generation is determined by a unitary process rather than a stochastic process. The unitary process, which is similar to a coefficient matrix $A$ in the linear equation $Y=A X$, means that it is deterministic and completely reversible. Therefore, even if we do not know the exact form of $T_{s 0 \rightarrow s 1}$ in Equation (2), we can analytically evaluate the equations without knowing the actual value of $T_{s 0 \rightarrow s 1}$. Thus, the quantum walk can still generate "all possible paths" without any prior assumption of probability distribution of each node. Although there seems to be a combinational explosion if we consider "all possible paths" all the time during the evolution, the "quantum collapse", which happened when the observation is applied, will map all the possible paths into a single determined path. We can then solve the combinatorial explosion problem in Procedure 2. With the solution of both Procedure 1 and Procedure 2, we can develop a simple calibration method to map the state vector to the traffic volumes as shown in Procedure 3.

With the above discussion, we introduced the quantum walk to simulate the random traffic fluctuation caused by a driver's random exit selection. By developing the quantum state vector representation of the driver's preference of exit, the quantum walk can be applied to simulate the dynamic updating process of random exit selection. With the relationship between the quantum state vector and the probability distribution for each choice, the probability of when a driver will leave the expressway through which exit can be generated. With the calibration of this simulated probability distribution to real observational traffic volume data, the traffic fluctuation caused by the driver's random exit selection can be generated. The integration of the deterministic part $F_{i k d}$ with the random part $F_{i k r}$ formulates our unified model, the Random Quantum Traffic Model (RQTM). The overall framework of RQTM is shown in Figure 2. 


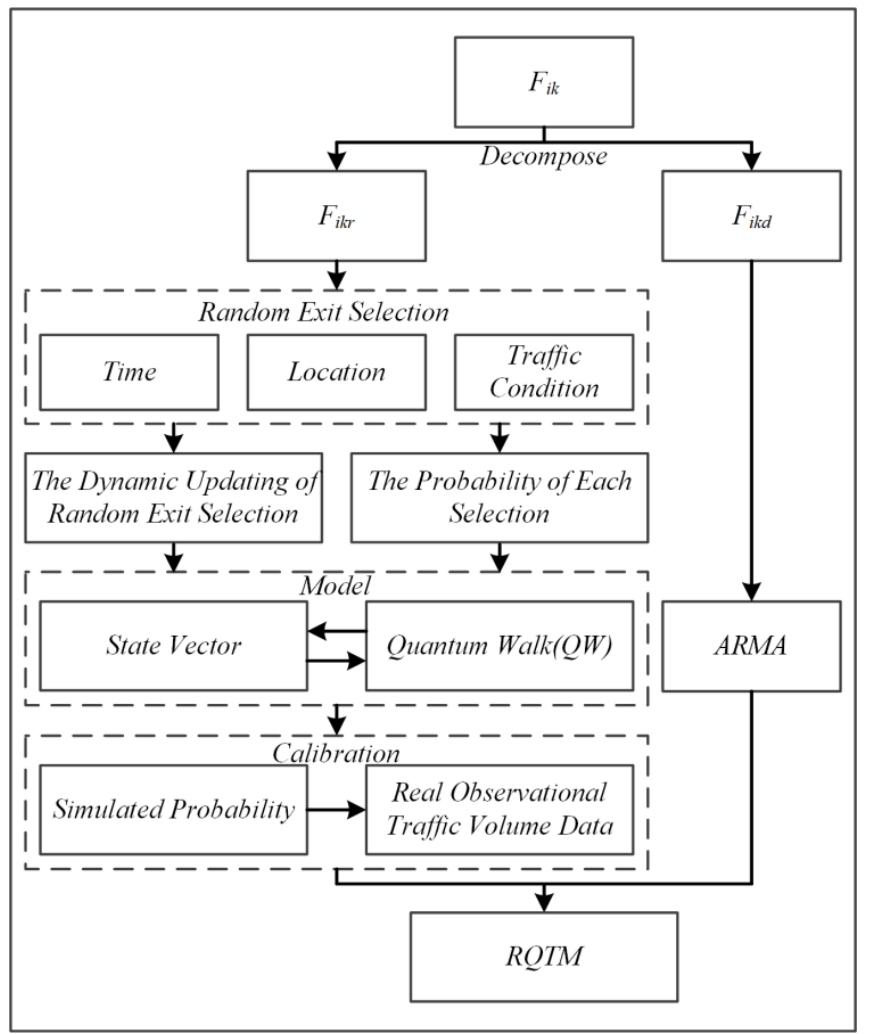

Figure 2. The overall framework.

\section{The RQTM Model}

\subsection{Quantum Walk Simulation of Random Exit Selection}

Suppose $G=(V, E)$ is the road network graph without considering its width, where $V$ is the set of nodes, which represents the possible choices at each exit, and $E$ is the set of edges, representing the possible evolution path of random exit selection over time. The number of nodes and edges in $G$ are $|V|$ and $|E|$, respectively. $A$ is the adjacency matrix of network $G$, that is

$$
A_{u v}=\left\{\begin{array}{l}
1, \text { if }(u, v) \in E \\
0, \text { otherwise }
\end{array}\right.
$$

Since different exits are connected by the expressway, the moving of drivers in the expressway can be modeled as transitions among different exits. At any time, a binary state indicating whether a driver will or will not exit the expressway (i.e., select or not select a node) can be constructed for each node. Since a driver will pick the travel route from multiple available routes based on considerations of economy, timeliness, and traffic conditions, although a driver has a deterministic destination, the exit that he or she selects can be random due to the multiplicity of available routes. The randomness of the exit selection can be modelled as the probability distribution of the nodes in the decision space.

Before we formulated the quantum walk, we first defined a Hilbert space $H \in C^{N}$, which is made up of a base state $|v\rangle$. The characteristic of a Hilbert space $H$ is that any quantum superposition state is a linear combination of base states, and the superposition state is also in $H$. Therefore, the state $|\varphi(k)\rangle$ of any driver with a quantum walk on $G$ can be defined as a linear superposition state of all base states, which is expressed as follows:

$$
|\varphi(k)\rangle=\sum_{v \in V} a_{v}(k)|v\rangle
$$

where $a_{v}(k)$ is the probability amplitude of the corresponding base state $|v\rangle$ at time $T_{k}$, $a_{v}(k) \in C$, and $\left|a_{v}(k)\right| \in[0,1]$. The probability of finding the walker at any exit at time $T_{k}$ is given by the squared modulus of the appropriate element of $\varphi(k)$ [36]. That is, the product 
of the probability amplitude and its complex conjugate is equivalent to the probability of the walker appearing on a node in the classical space. Therefore, the probability that a driver is in a base state $|v\rangle$ at time $T_{k}$ can be expressed as

$$
p(|v\rangle, k)=\alpha_{v}(k) \alpha_{v}^{*}(k)
$$

where $\alpha_{v}^{*}(k)$ is a complex conjugate of $\alpha_{v}(k)$. At time $T_{k}$, it satisfies $\sum_{v \in V} p(|v\rangle, k)=1$.

Unlike the classical random walk, the process of the quantum walk is not a Markov chain [33]. The evolution of state vector $|\varphi(k)\rangle$ with time $T_{k}$ is realized by the following unitary transformation:

$$
\frac{d}{d t}|\varphi(k)\rangle=i A|\varphi(k)\rangle
$$

It can be seen from Equation (8) that the state evolution process depends on the adjacency matrix $A$. However, as a differential equation with complex coefficients, it is difficult to solve it directly. Therefore, it can be transformed into a matrix form from the discrete point of view, and the quantum walk can be efficiently solved by matrix operation.

Assuming a driver starts at the node $|v\rangle$, from Equation (8), we know that it will have a state vector

$$
|\varphi(k)\rangle=e^{-i A}|v\rangle
$$

Then the probability $p_{v u}(k)$, indicating the walker moves from node $v$ to node $u$, is

$$
\left.p_{v u}(k)=|| \mu \| \varphi(k)\right\rangle\left.\right|^{2}
$$

Analogously to Equation (3), we can express the state vector as a matrix

$$
\left(\begin{array}{c}
\varphi_{1}{ }^{\prime} \\
\vdots \\
\varphi_{n}{ }^{\prime}
\end{array}\right)=\left(\begin{array}{ccc}
\mu_{11} & \cdots & \mu_{1 n} \\
\vdots & \ddots & \vdots \\
\mu_{n 1} & \cdots & \mu_{n n}
\end{array}\right) \cdot\left(\begin{array}{c}
\varphi_{1} \\
\vdots \\
\varphi_{1}
\end{array}\right)
$$

where $u$ is a unitary matrix varying with time. Similar to Equations (10) and (12), we can construct a time evolution operator $U(k)=e^{-i A k}$ to construct the dynamic evolution quantum walk [30]. As the topology of the expressway is a line, we have

$$
A=\left[\begin{array}{cccccc}
0 & 1 & 0 & \ldots & 0 & 0 \\
1 & 0 & 0 & \ldots & 0 & 0 \\
0 & 1 & 0 & \ldots & 0 & 0 \\
\vdots & \vdots & \vdots & \ddots & \vdots & \vdots \\
0 & 0 & 0 & \cdots & 1 & 0 \\
0 & 0 & 0 & \cdots & 0 & 1 \\
0 & 0 & 0 & \cdots & 0 & 0
\end{array}\right]
$$

As $A$ is a matrix, and the numerical solution of such a model is complex, we can use a polynomial expansion to approximate the quantum walk [31]. From Equation (9), we have

$$
e^{-i A k}=\sum_{n=0}^{N-1} c_{n} A^{n}
$$

where $N$ is the number of distinct eigenvalues of $A$, and $c_{n}$ are unknown coefficients that must be determined. These coefficients can be fixed by using Equation (13) and continue to be valid when $A$ is replaced by each of its eigenvalues. Assuming the matrix exponential of $U(k)$ as

$$
U(k)=c_{0} I+c_{1} A+c_{2} A^{2}+c_{3} A^{3}+\cdots+c_{n} A^{n}
$$

where $A$, which can be seen as $A^{0}$, is the identity matrix, and $c_{n}$ is the weight coefficients to be determined. Our evaluation of the time evolution operator is based on the 
Cayley-Hamilton theorem [36], which states that every square matrix satisfies its own characteristic equation

$$
\operatorname{det}(A-I \lambda)=0
$$

where $A$ is the original matrix, $I$ is the identity matrix, and $\lambda$ is the eigenvalue. The characteristic equation is a polynomial equation in $\lambda$ and will remain valid when $\lambda$ is replaced by $A$. With the Cayley-Hamilton theorem, we can replace the Hamiltonian with each of its eigenvalues in the previous equation to obtain the following system of equations:

$$
\left[\begin{array}{c}
e^{-i \lambda_{1} k} \\
e^{-i \lambda_{2} k} \\
\vdots \\
e^{-i \lambda_{n-1} k} \\
e^{-i \lambda_{n} k}
\end{array}\right]=\left[\begin{array}{ccccc}
1 & \lambda_{1} & \lambda_{1}{ }^{2} & \cdots & \lambda_{1}{ }^{n-1} \\
1 & \lambda_{2} & \lambda_{2}{ }^{2} & \cdots & \lambda_{1}{ }^{n-1} \\
\vdots & \vdots & \ddots & \cdots & \vdots \\
1 & \lambda_{n-1} & \lambda_{n-1}{ }^{2} & \cdots & \lambda_{n-1}{ }^{n-1} \\
1 & \lambda_{n} & \lambda_{n}{ }^{2} & \cdots & \lambda_{n}{ }^{n-1}
\end{array}\right]\left[\begin{array}{c}
c_{1} \\
c_{2} \\
\vdots \\
c_{n-1} \\
c_{n}
\end{array}\right]
$$

One can solve for the coefficients with simple linear algebra to obtain an expression for the time evolution operator: a $n \times n$ matrix. With this matrix solution, we can then solve Equation (10) to obtain the quantum walk simulation. With Equation (16), we can have the dynamic change of probability of each node. Therefore, the dynamics of random exit selection can be simulated.

\subsection{Map Probability Dynamics to Traffic Volumes}

In the quantum walk-based expressway simulation model, there are two key parameters that need to be determined. One is the time step $\Delta k$ of the quantum walk evolution, and the other is the correction factor $a_{i}$ from the probability magnitude to the real traffic data. Assuming that the time series of the traffic flow of the exit obtained by the real observation is $F_{i k}$, and the quantum walk of the parameter is $\Delta k$, the $i$ th-exit probability sequence of the model output is $P_{i}(k)$, then the quantum walk correction model can be constructed as follows:

$$
F_{i k}=a_{i} P_{i}(k)
$$

In the above model, $P_{i}(k)$ is a probability determined by the characteristics of the quantum walk. The variable $k$ determines the characteristics and laws of the quantum walk probability evolution, which determines the time scale and period characteristics of the quantum walk simulation data (see the discussion in Section 5). The parameterization is carried out by means of stepwise approximation. Referring to the determination method of the link coefficient in the classical random walk, we can gradually increase $k$ with an interval of $\Delta k$ and compare different simulation results with the measured data in the overall waveform structure and statistics. The best match will produce the optimal $\Delta k$. After the optimal $\Delta k$ parameter is determined, the correction coefficient $a_{i}$ can be obtained by directly fitting the simulation results to the real observation using the least square method.

As there is a large amount of regular traffic that periodically changes in the traffic flow, which cannot be captured by the quantum walk, we also used the autoregressive moving average model to model the residual series.

The autoregressive moving average model is composed of the autoregressive model and the moving average model, which is an important method to study time series, and the corresponding parameters are $p$ and $q$, respectively. The $p$ denotes the lag number of the time series data used in the prediction model, expressed as the partial autocorrelation coefficient, which is close to 0 in the $p$-order lag, also called the AR/auto regressive term. The $q$ represents the prediction used in the moving average prediction model. The hysteresis of the error is expressed as the autocorrelation coefficient approaching 0 at the $q$-order lag, also known as the MA/moving average term. The overall formula of the autoregressive moving average model is

$$
y_{t}=\mu+\phi_{1} \times y_{k-1}+\cdots+\phi_{p} \times y_{k-p}+\theta_{1} \times \varepsilon_{k-1}+\cdots+\theta_{p} \times \varepsilon_{k-p}
$$


where $\phi$ is the coefficient of the auto regressive process, and $\theta$ is the coefficient of the moving average process.

To summarize, the overall model of RQTM is

$$
\operatorname{RQTM}\left(F_{i k}\right)=Q W(k)+\operatorname{ARMA}(p, q)=\alpha_{i} P(i, k)+\operatorname{ARMA}(p, q)
$$

where $i, k$ are the indices of exit and time, respectively, $\alpha_{i}$ is the correction factor from the probability $P(i, k)$ to the real traffic data, and $p$ and $q$ are the fitting parameter of ARMA.

\section{Experiments}

\subsection{Research Data and Experiment Configuration}

The Shanghai-Nanjing Expressway has one of the busiest traffic flows with complicated conditions in eastern China [37]. Since the trend of "same city" in the Yangtze River Delta area is very obvious, this paper selected the Nanjing-Changzhou section of the Shanghai-Nanjing Expressway as the study area and selected the distribution of vehicles departing from Nanjing and passing through 7 exits (Figure 3) during the period from 1 December 2015 to 30 December 2015. To form the intercity expressway traffic, we only selected the vehicles entering directly from Nanjing at each exit. The original data contains information about each vehicle passing through the exit, so the volume is huge. To reduce the data volume, we accumulated the number of vehicles that passed each exit in one hour. Then, we had the hourly time series for each exit. The overall sample points for each exit are 31 (days) $\times 24$ (hours) $=744$ (time points).

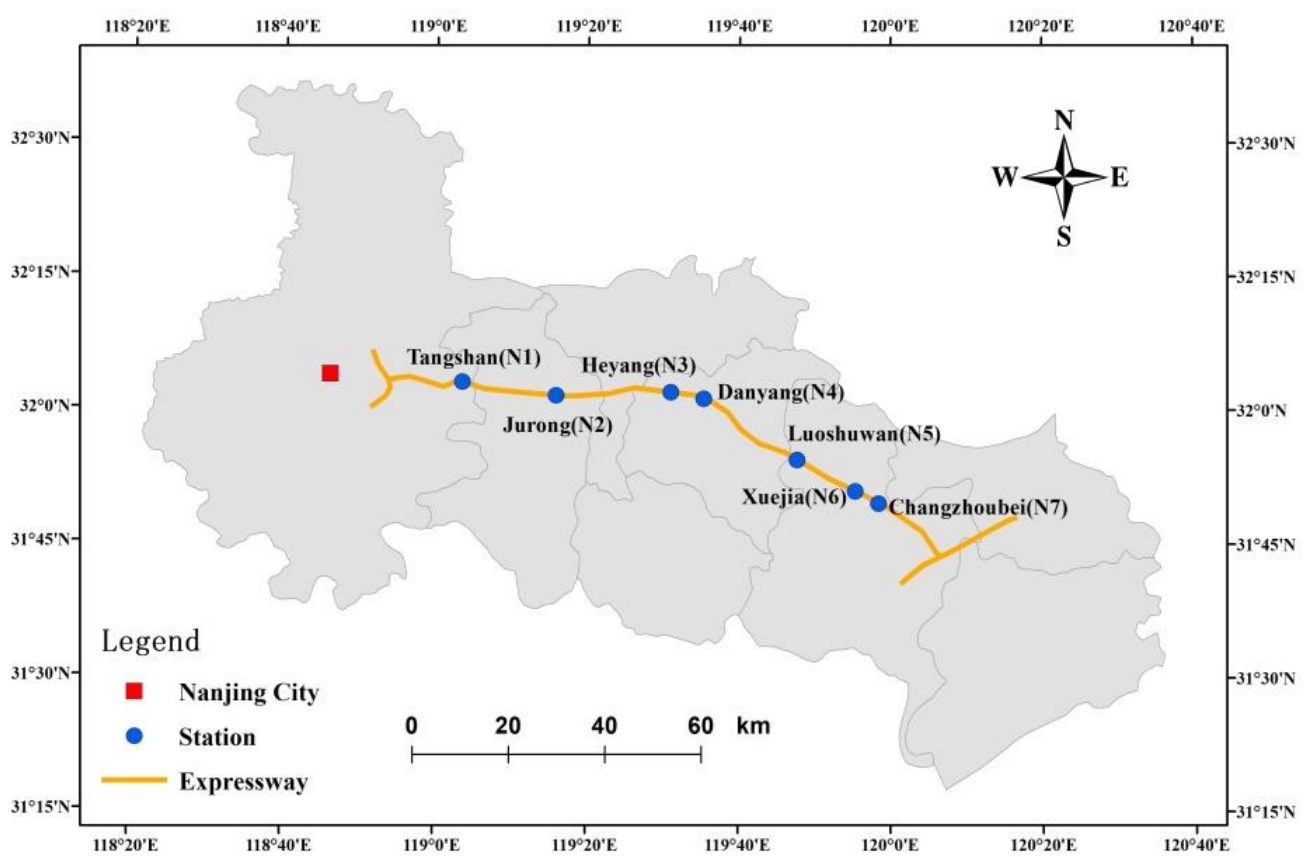

Figure 3. Research area and exit distribution.

Based on the distribution of each exit in the original data and the road topology, the adjacency matrix between each exit was constructed. The quantum walk simulation experiment was conducted based on the adjacency matrix. The control parameters of the quantum walk were mainly $\Delta k$ and $\alpha_{i}$, which determined the time interval of the simulated unit time through the exit of the vehicle, thus affecting the time scale characteristics of the simulated time series (length and number of cycles, etc.) over a long time period. To this end, we traversed different $\Delta k$ ways to obtain the value of the optimal control parameter $\Delta k$ (for a detailed analysis, see discussion in Section 5). For this, $\Delta k$ was gradually increased from 0 to 1 at the interval of 0.01 , by comparing the statistics of different $\Delta k$ values, the best value of $\Delta k$ was determined. The probability sequence obtained by the simulation 
was subjected to calibration, which was mapped into the total sequence of real observation data; the model performance and statistics were calculated, and the residual analysis was performed. To further demonstrate the performance of the quantum walk, the classical random walk method was used at the same time for comparison. The connection degree of the classical random walk was also determined by the traverse method. In order to further reveal the regularity of the residual part, the autoregressive moving average model was used to fit the residual sequence. Finally, the whole experiment was completed to verify the RQTM.

For the performance of RQTM, this paper used three statistical indicators to evaluate it, including the mean absolute error (MAE), the root mean square error (RMSE), and the coefficient of determination $\left(\mathrm{R}^{2}\right)$. The definition of these statistical indicators is listed in Table 1 . In Table $1, y_{i}$ is the real observation data, $\overline{y_{i}}$ is the average of the real observation data, and $\hat{y}_{i}$ is the modeling data.

Table 1. Model performance evaluation indicators.

\begin{tabular}{cc}
\hline Statistical Index & Definition \\
\hline Mean Absolute Error (MAE) & $M A E=\frac{1}{m} \sum_{i=1}^{m}\left|y_{i}-\hat{y}_{i}\right|$ \\
Root Mean Square Error (RMSE) & $R M S E=\sqrt{\frac{1}{m} \sum_{i=1}^{m}\left(y_{i}-\hat{y}_{i}\right)^{2}}$ \\
Coefficient of Determination $\left(\mathrm{R}^{2}\right)$ & $R^{2}=1-\sum_{i=1}^{m}\left|y_{i}-\hat{y}_{i}\right|^{2} / \sum_{i=1}^{m}\left|y_{i}-\overline{y_{i}}\right|^{2}$ \\
\hline
\end{tabular}

\subsection{Results}

Based on the experiment configuration, the average statistics of the quantum walk simulation with different $\Delta k$ values are listed in Table 2 , and when $\Delta k=0.13$, the statistics were the best, so the optimal fitting parameter $\Delta k$ of quantum walk for the seven exits was set to 0.13 . Besides, the coefficients of RQTM are listed in Table 3. The $\alpha_{i}$ coefficient for seven exits was between 25.23 and 503.42, which is proportional to the total number of vehicles passing through each exit. For the coefficients of the autoregressive moving average model for the regularity part $F_{i k d}$, it is obvious that the autoregressive coefficient of the quantum walk residual series modeling has same the coefficients of $p=2$, except $\mathrm{N} 3$, which is $p=3$. This indicates a significant time correlation of $2 \mathrm{~h}$ in the fluctuation structure of vehicle flow series, which is consistent with the situation that the travel time from Nanjing to Changzhou is less than $2 \mathrm{~h}$. From the coefficient of the moving average coefficient, the moving average coefficients $q$ of the quantum walk modeling residuals are larger than the $q$ of the classical random walk modeling residuals, indicating that the residual of the quantum walk modeling is more stationary than that of the classical random walk modeling.

Table 2. Average statistics of quantum walk simulation with different $\Delta k$.

\begin{tabular}{ccc}
\hline $\boldsymbol{\Delta} \boldsymbol{k}$ & MAE & RMSE \\
\hline 0.1 & 25.04 & 32.86 \\
0.13 & 23.62 & 31.28 \\
0.2 & 25.23 & 32.74 \\
0.3 & 25.34 & 32.81 \\
\hline
\end{tabular}

The simulation results based on the RQTM are shown in Figure 4, respectively. We found that the RQTM reproduces real observation data well. The distribution of peaks and troughs obtained in different time periods is consistent with the law of quasi-periodic fluctuation found in the traffic origin-destination matrix in recent studies [38,39]. The statistics of the model performance of the RQTM and "RW + ARMA" are listed in Table 4, and the $\mathrm{R}^{2}$ of RQTM ranged from 0.5 (N5) to 0.85 (N1). Compared with the "RW + ARMA" 
model, the increment of $\mathrm{R}^{2}$ is from $21.28 \%$ (N7) to $104.98 \%$ (N3), and the decrement of the RMSE is from $11.61 \%$ (N2) to $32.92 \%(\mathrm{~N} 1)$, and the decrement of MAE is from $13.42 \%(\mathrm{~N} 2)$ to $30.34 \%$ (N1). In general, the RQTM can model the traffic flow of each exit well.

Table 3. The coefficients of RQTM.

\begin{tabular}{|c|c|c|c|}
\hline Exits & Methods & $\alpha_{i}$ & $\begin{array}{c}\text { ARMA } \\
\operatorname{Model}(p, q)\end{array}$ \\
\hline \multirow{2}{*}{ N1 } & RQTM & 503.42 & ARMA $(2,5)$ \\
\hline & "RW + ARMA" & 279.91 & ARMA $(5,2)$ \\
\hline \multirow{2}{*}{$\mathrm{N} 2$} & RQTM & 33.56 & $\operatorname{ARMA}(2,2)$ \\
\hline & "RW + ARMA" & 16.96 & ARMA $(3,1)$ \\
\hline \multirow{2}{*}{ N3 } & RQTM & 71.19 & ARMA $(3,1)$ \\
\hline & "RW + ARMA" & 47.75 & ARMA $(1,1)$ \\
\hline \multirow{2}{*}{$\mathrm{N} 4$} & RQTM & 80.28 & ARMA $(2,3)$ \\
\hline & "RW + ARMA" & 69.03 & ARMA $(2,2)$ \\
\hline \multirow{2}{*}{ N5 } & RQTM & 25.23 & $\operatorname{ARMA}(2,3)$ \\
\hline & "RW + ARMA" & 16.15 & $\operatorname{ARMA}(2,3)$ \\
\hline \multirow[b]{2}{*}{ N6 } & RQTM & 75.91 & ARMA $(2,3)$ \\
\hline & "RW + ARMA" & 51.00 & ARMA $(5,1)$ \\
\hline \multirow{2}{*}{ N7 } & RQTM & 38.81 & $\operatorname{ARMA}(2,5)$ \\
\hline & "RW + ARMA" & 34.85 & ARMA $(2,2)$ \\
\hline
\end{tabular}

Table 4. Statistics of the model performance of RQTM and "RW + ARMA".

\begin{tabular}{ccccc}
\hline Exits & Methods & MAE & RMSE & $\mathbf{R}^{\mathbf{2}}$ \\
\hline \multirow{2}{*}{$\mathrm{N} 1$} & RQTM & 24.84 & 34.71 & 0.85 \\
& "RW + ARMA" & 35.66 & 89.32 & 0.67 \\
\hline \multirow{2}{*}{$\mathrm{N} 2$} & RQTM & 3.33 & 4.76 & 0.57 \\
& "RW + ARMA" & 3.91 & 5.66 & 0.45 \\
\hline \multirow{2}{*}{ N3 } & RQTM & 5.17 & 7.11 & 0.66 \\
& "RW + ARMA" & 7.39 & 13.44 & 0.32 \\
\hline \multirow{2}{*}{ N4 } & RQTM & 7.93 & 10.95 & 0.76 \\
& "RW + ARMA" & 11.13 & 17.29 & 0.55 \\
\hline \multirow{2}{*}{ N5 } & RQTM & 2.80 & 3.77 & 0.50 \\
& "RW + ARMA" & 3.30 & 4.85 & 0.34 \\
\hline \multirow{2}{*}{ N6 } & RQTM & 7.00 & 9.43 & 0.77 \\
& "RW + ARMA" & 8.95 & 14.50 & 0.61 \\
\hline \multirow{2}{*}{ N7 } & RQTM & 4.06 & 5.46 & 0.68 \\
& "RW + ARMA" & 4.69 & 9.80 & 0.56 \\
\hline
\end{tabular}




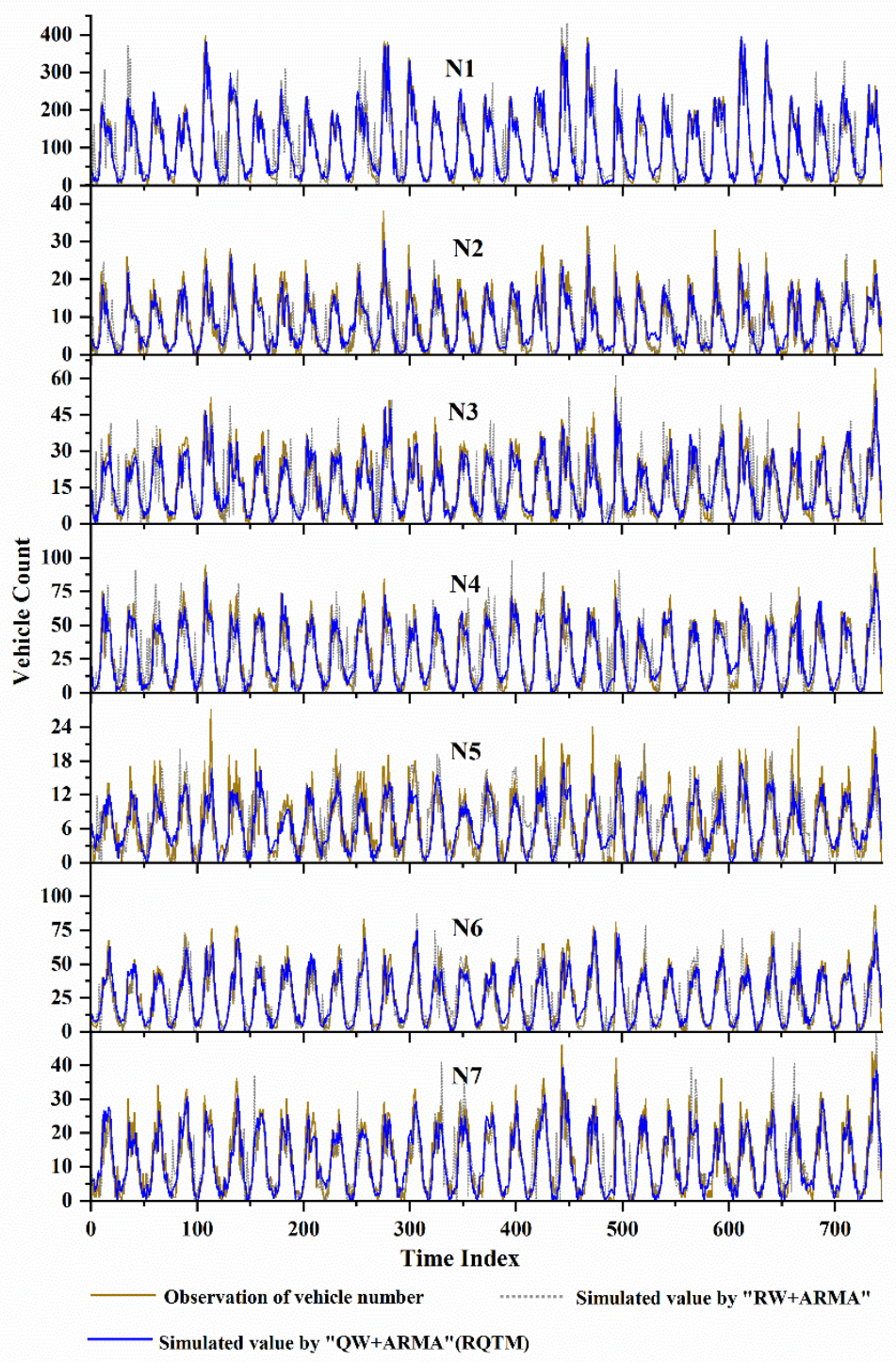

Figure 4. The simulation results of RQTM.

\section{Discussion}

\subsection{Improvement of Modeling $F_{i k r}$ over Classical Random Walk}

In this section, we provide a detailed comparison of the modeling of the random exit selection using the quantum walk and using the classical random walk through three typical exits (N1, N3 and N7). The comparison results of the quantum walk and classical random walk are shown in Figure 5. From Figure 5, it is clear that the amplitude of the quantum walk simulation varies in a quasi period, which fits much better with the observation data. Firstly, there are more high-frequency fluctuations in the classical random walk simulation results, such as 0 to 50, 125 to 150, and 430 to 470 time intervals of the N1 and N3 exits, which have obvious scale differences with the real observation data. However, the quantum walk has no such high-frequency, random fluctuations. Secondly, the distribution of the peaks and troughs simulated by the quantum walk is relatively uniform without obvious aggregation. For the three exits, the total number of peaks of the actual observation data is 31 peaks for each exit, and the total number of peaks with good fitting performance of the quantum walk and classical random walk is 25 and 16, respectively, so the corresponding proportion is 31:25:16. Therefore, the fitting performance of the quantum walk on peaks is better than that of the classical random walk. Thirdly, because the quantum walk only simulates the randomness of the expressway traffic flow, it is normal that there is a certain 
deviation between the simulated value and the real observation data. However, compared with the classical random walk, the simulation results of the quantum walk are more consistent with the distribution trend of actual data, which can better recover the change trend of the expressway traffic flow. In conclusion, compared with the classical random walk, the quantum walk can better reveal the randomness of the expressway traffic flow.

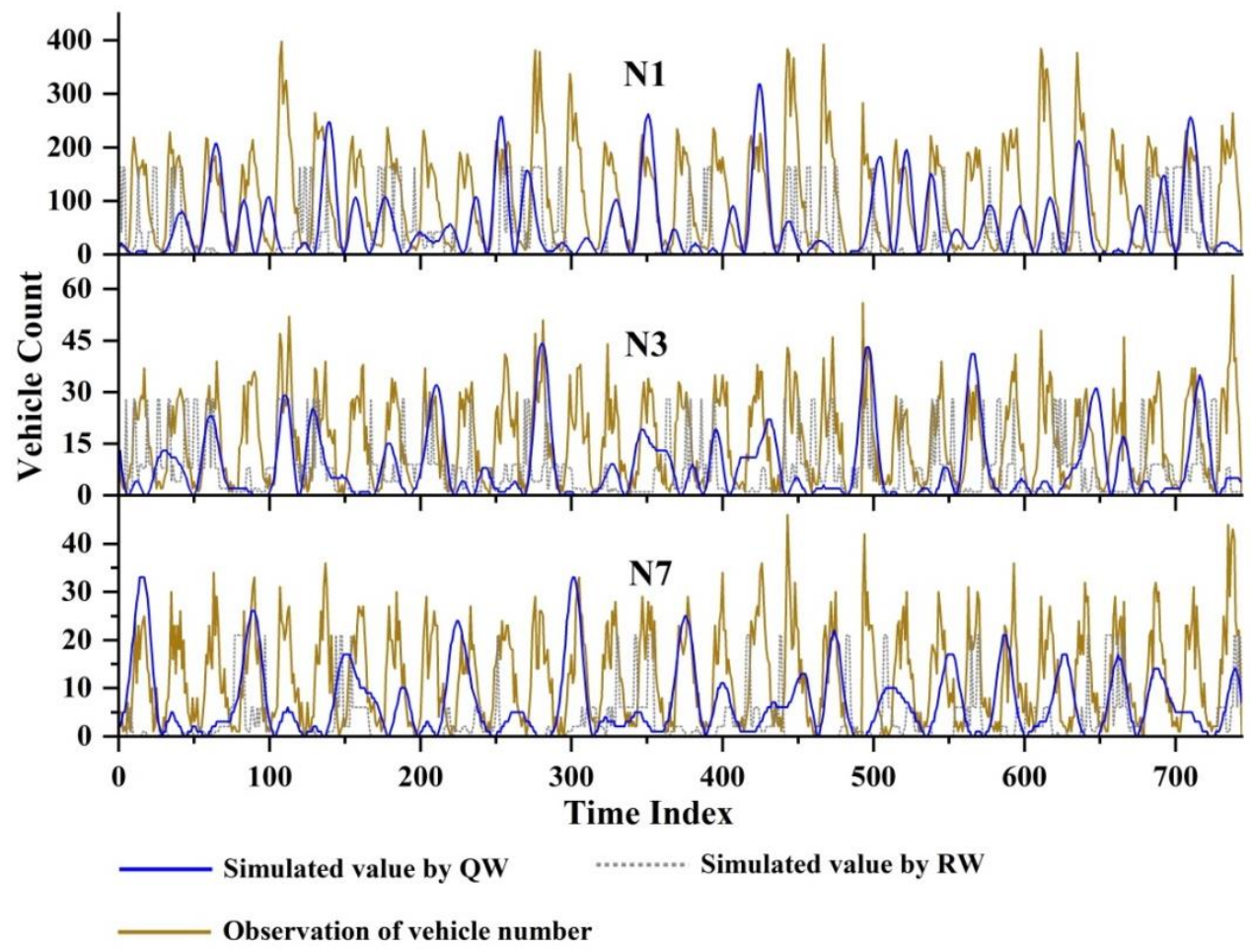

Figure 5. Comparison between simulation results and real observation data.

\subsection{Applicable Issues and Future Works of Quantum Walk}

Due to the ideal abstraction of the expressway traffic, our RQTM model still has large potential to be improved from the following aspects. Firstly, the research data used in this paper is hour-scale traffic flow observation data. It did not use traffic data at high temporal resolution, such as minute-scale or even individual vehicle trajectory. The vehicle type was also not used. In future applications, more detailed expressway traffic flow data can be considered, which might improve the modeling accuracy. Secondly, we only considered the single lane in the RQTM, the lane change situation was not considered. Integrating lane change information may also improve the performance of the RQTM. Thirdly, this study did not consider the differences in spatial distance between different exits. The spatial distances between different exits would have an impact on the driver's random exit selection. In the candidate exits of the driver, most of these exits will not be too far away from their destinations where the random decision occurs, particularly when the traffic is heavy. In a similar process, the spatial distance between exits will directly affect the probability of the driver exiting the expressway traffic flow, which needs to be weighted in the quantum walk. In this work, we manly concerned ourselves with the randomness of the exit selection due to the random travel route decision. The precise decision-making data of drivers will help to improve the simulation accuracy of the proposed model. Thus, we will conduct in-depth research through video monitoring and other means in future research.

\section{Conclusions}

This paper developed a RQTM model in an effort to model the effects of the random exit selection of drivers on intercity expressway traffic flow. The RQTM models the deterministic part with an autoregressive moving average model and the randomness caused 
by the random exit selection of drivers with the quantum walk. For the seven exits, the coefficients of determination of the RQTM ranged from 0.5 to 0.85 , with an increment of $21.28 \%$ to $104.98 \%$ compared with the classical random walk and the ARMA model. Meanwhile, the RMSE decreased by $11.61 \%$ to $32.92 \%$. The RQTM can better reproduce the stochastic fluctuations caused by random decision making. Our method provides a good potential for modeling the unobservable driver's random exit selection on traffic flow simulation.

Author Contributions: Conceptualization, D.L. and Z.Y.; methodology and validation X.H.; formal analysis and investigation, X.Z.; resources, Z.Y.; writing-original draft preparation, D.L. and Z.Y.; writing-review and editing, W.L. and A.X.Z.; supervision, D.L.; funding acquisition, D.L. and Z.Y. All authors have read and agreed to the published version of the manuscript.

Funding: This research was funded by the National Natural Science Foundation of China (No. 41971404 and 42001320), the Postdoctoral Science Foundation of China (No. 2021M702757) and the Priority Academic Program Development of Jiangsu Higher Education Institutions (PAPD).

Institutional Review Board Statement: Not applicable.

Informed Consent Statement: Not applicable.

Data Availability Statement: Not applicable.

Conflicts of Interest: The authors declare no conflict of interest.

\section{References}

1. Jiang, R.; Wu, Q.; Zhu, Z. Full velocity difference model for a car-following theory. Phys. Rev. E 2001, 64, 17101. [CrossRef] [PubMed]

2. Ramezani, M.; Geroliminis, N. On the estimation of arterial route travel time distribution with Markov chains. Transp. Res. Part B Methodol. 2012, 46, 1576-1590. [CrossRef]

3. Ermagun, A.; Levinson, D. Spatiotemporal traffic forecasting: Review and proposed directions. Transp. Rev. 2018, 38, 786-814. [CrossRef]

4. Seo, T.; Bayen, A.M.; Kusakabe, T.; Asakura, Y. Traffic state estimation on highway: A comprehensive survey. Annu. Rev. Control 2017, 43, 128-151. [CrossRef]

5. Bekiaris-Liberis, N.; Roncoli, C.; Papageorgiou, M. Highway traffic state estimation with mixed connected and conventional vehicles. IEEE Trans. Intell. Transp. Syst. 2016, 17, 3484-3497. [CrossRef]

6. Chen, R.B. Models of Count with Endogenous Choices. Transp. Res. Procedia 2017, 23, 460-479. [CrossRef]

7. Bekhor, S.; Prashker, J.N. GEV-based destination choice models that account for unobserved similarities among alternatives. Transp. Res. Part B Methodol. 2008, 42, 243-262. [CrossRef]

8. Danial, A.; Ali, A.A.; Mohammad, S. Prediction of vessels locations and maritime traffic using similarity measurement of trajectory, Ann. GIS 2021, 27, 151-162. [CrossRef]

9. Tolouei, R.; Psarras, S.; Prince, R. Origin-Destination trip matrix development: Conventional methods versus mobile phone data. Transp. Res. Procedia 2017, 26, 39-52. [CrossRef]

10. Yuan, Y.; Van Lint, J.W.C.; Wilson, R.E.; Van Wageningen-Kessels, F.; Hoogendoorn, S.P. Real-time lagrangian traffic state estimator for freeways. IEEE Trans. Intell. Transp. Syst. 2012, 13, 59-70. [CrossRef]

11. Xu, C.; Li, Z.; Wang, W. Short-term traffic flow prediction using a methodology based on autoregressive integrated moving average and genetic programming. Transport 2016, 31, 343-358. [CrossRef]

12. Haddad, J.; Ramezani, M.; Geroliminis, N. Cooperative traffic control of a mixed network with two urban regions and a freeway. Transp. Res. Part B Methodol. 2013, 54, 17-36. [CrossRef]

13. Adacher, L.; Tiriolo, M. A macroscopic model with the advantages of microscopic model: A review of Cell Transmission Model's extensions for urban traffic networks. Simul. Model. Pract. Theory 2018, 86, 102-119. [CrossRef]

14. Barlovic, R.; Schadschneider, A.; Schreckenberg, M. Random walk theory of jamming in a cellular automaton model for traffic flow. Phys. A Stat. Mech. Its Appl. 2001, 294, 525-538. [CrossRef]

15. González, M.C.; Hidalgo, C.A.; Barabási, A.L. Understanding individual human mobility patterns. Nature 2008, 453, 779-782. [CrossRef]

16. Szell, M.; Sinatra, R.; Petri, G.; Thurner, S.; Latora, V. Understanding mobility in a social petri dish. Sci. Rep. 2012, 2, 457. [CrossRef]

17. Zhu, J.; Ye, X. Development of destination choice model with pairwise district-level constants using taxi GPS data. Transp. Res. Part C Emerg. Technol. 2018, 93, 410-424. [CrossRef]

18. Hasnat, M.M.; Faghih-Imani, A.; Eluru, N.; Hasan, S. Destination choice modeling using location-based social media data. J. Choice Model. 2019, 31, 22-34. [CrossRef] 
19. Qu, X.; Zhang, J.; Wang, S. On the stochastic fundamental diagram for freeway traffic: Model development, analytical properties, validation, and extensive applications. Transp. Res. Part B Methodol. 2017, 104, 256-271. [CrossRef]

20. Gao, S.; Li, M.; Liang, Y.; Marks, J.; Kang, Y.; Li, M. Predicting the spatiotemporal legality of on-street parking using open data and machine learning. Ann. GIS 2019, 25, 299-312. [CrossRef]

21. Calabrese, F.; Colonna, M.; Lovisolo, P.; Parata, D.; Ratti, C. Real-time urban monitoring using cell phones: A case study in Rome. IEEE Trans. Intell. Transp. Syst. 2011, 12, 141-151. [CrossRef]

22. Song, C.; Koren, T.; Wang, P.; Barabási, A.-L. Modelling the scaling properties of human mobility. Nat. Phys. 2010, 6, 818-823. [CrossRef]

23. Zhao, X.; Wan, C.; Sun, H.; Xie, D.; Gao, Z. Dynamic rerouting behavior and its impact on dynamic traffic patterns. IEEE Trans. Intell. Transp. Syst. 2017, 18, 2763-2779. [CrossRef]

24. Zeng, G.; Li, D.; Guo, S.; Gao, L.; Gao, Z.; Stanley, H.E.; Havlin, S. Switch between critical percolation modes in city traffic dynamics. Proc. Natl. Acad. Sci. USA 2019, 116, 23-28. [CrossRef] [PubMed]

25. Simini, F.; González, M.C.; Maritan, A.; Barabási, A.-L. A universal model for mobility and migration patterns. Nature 2012, 484, 96-100. [CrossRef] [PubMed]

26. Yan, X.-Y.; Wang, W.-X.; Gao, Z.-Y.; Lai, Y.-C. Universal model of individual and population mobility on diverse spatial scales. Nat. Commun. 2017, 8, 1639. [CrossRef] [PubMed]

27. Sheu, J.B. Characterisation of driver behaviour during car following using quantum optical flow theory. Transp. A Transp. Sci. 2013, 9, 269-298. [CrossRef]

28. Biamonte, J.; Faccin, M.; De Domenico, M. Complex networks from classical to quantum. Commun. Phys. 2019, 2, 53. [CrossRef]

29. Linke, N.M.; Maslov, D.; Roetteler, M.; Debnath, S.; Figgatt, C.; Landsman, K.A.; Wright, K.; Monroe, C. Experimental comparison of two quantum computing architectures. Proc. Natl. Acad. Sci. USA 2017, 114, 3305-3310. [CrossRef]

30. Childs, A.M.; Gosset, D.; Webb, Z. Universal computation by multiparticle quantum walk. Science 2013, 339, 791-794. [CrossRef]

31. Tsuji, Y.; Estrada, E.; Movassagh, R.; Hoffmann, R. Quantum Interference, Graphs, Walks, and Polynomials. Chem. Rev. 2018, 118, 4887-4911. [CrossRef] [PubMed]

32. Venegas-Andraca, S.E. Quantum walks: A comprehensive review. Quantum Inf. Process. 2012, 11, 1015-1106. [CrossRef]

33. Ellinas, D.; Konstandakis, C. Parametric Quantum Search Algorithm as Quantum Walk: A Quantum Simulation. Rep. Math. Phys. 2016, 77, 105-128. [CrossRef]

34. Martinez-Martinez, I.; Sanchez-Burillo, E. Quantum stochastic walks on networks for decision-making. Sci. Rep. 2016,6 , 23812. [CrossRef]

35. Altmann, Y.; McLaughlin, S.; Padgett, M.J.; Goyal, V.K.; Hero, A.O.; Faccio, D. Quantum-inspired computational imaging. Science 2018, 361, eaat2298. [CrossRef]

36. Tödtli, B.; Laner, M.; Semenov, J.; Paoli, B.; Blattner, M.; Kunegis, J. Continuous-time quantum walks on directed bipartite graphs. Phys. Rev. A 2016, 94, 052338. [CrossRef]

37. Gao, J.; Yuan, F. Economic transition, firm dynamics, and restructuring of manufacturing spaces in urban China: Empirical evidence from Nanjing. Prof. Geogr. 2017, 69, 504-519. [CrossRef]

38. Chen, D.; Laval, J.; Zheng, Z.; Ahn, S. A behavioral car-following model that captures traffic oscillations. Transp. Res. Part B Methodol. 2012, 46, 744-761. [CrossRef]

39. Bauer, D.; Richter, G.; Asamer, J.; Heilmann, B.; Lenz, G.; Kölbl, R. Quasi-Dynamic Estimation of OD Flows from Traffic Counts Without Prior OD Matrix. IEEE Trans. Intell. Transp. Syst. 2018, 19, 2025-2034. [CrossRef] 